\title{
EchoGéo
}

L'Asie centrale : le temps des recompositions

\section{Les souvenirs du passeur solitaire}

Entretien avec Jean-Pierre Raison. Propos recueillis par Jean Marie Théodat

Jean Marie Théodat et Jean-Pierre Raison

\section{OpenEdition}

\section{Journals}

Édition électronique

URL : https://journals.openedition.org/echogeo/11297

DOI : 10.4000/echogeo. 11297

ISSN : 1963-1197

Éditeur

Pôle de recherche pour l'organisation et la diffusion de l'information géographique (CNRS UMR 8586)

Référence électronique

Jean Marie Théodat et Jean-Pierre Raison, « Les souvenirs du passeur solitaire », EchoGéo [En ligne], 9 | 2009, mis en ligne le 04 juillet 2009, consulté le 03 août 2021. URL : http://journals.openedition.org/ echogeo/11297; DOI : https://doi.org/10.4000/echogeo.11297

Ce document a été généré automatiquement le 3 août 2021

EchoGéo est mis à disposition selon les termes de la licence Creative Commons Attribution - Pas d'Utilisation Commerciale - Pas de Modification 4.0 International (CC BY-NC-ND) 


\title{
Les souvenirs du passeur solitaire
}

\author{
Entretien avec Jean-Pierre Raison. Propos recueillis par Jean Marie \\ Théodat
}

Jean Marie Théodat et Jean-Pierre Raison

1 Passée la grille qui sépare la cour de plain-pied avec le trottoir dallé de frais, on pourrait se croire dans la cour de l'église, sur le seuil du presbytère, sur le point de rendre visite au curé, tant l'atmosphère recluse de ce refuge contraste avec l'animation brouillonne du quartier. D'une façon presque rituelle, je sens le poids de la pierre en passant sous le linteau voûté qui donne sur l'escalier étroit. Je néglige l'ascenseur et me glisse dans ce colimaçon de pierre et de chêne, les marches vénérables grinçant sous mes pas. Arrivé au premier étage, le dôme de l'église Saint-Paul se dévoile au détour d'une fenêtre palière dont la vitre bossuée de bulles fait gondoler l'ardoise et tanguer les gouttières qui bordent les toits. Je sonne à la porte à l'heure moins cinq de notre rendez-vous.

2 Pour l'avoir eu comme professeur, je ne puis pourtant pas prétendre au titre de disciple, mais c'est avec le même respect, mitigé de terreur sacrée, que je lui présente le projet : un retour réflexif sur son parcours de géographe. Il acquiesce à la condition que cette causerie débouche sur une objectivation du discours devant nous conduire, non à un déroulé d'anecdotes sans suite, mais à une mise en perspective du métier de géographe, pour en comprendre les enjeux et les attentes.

3 Il sert lui-même le café. Si serré que j'en bois à peine une goutte. Il devine mon désarroi, ouvre sans délai le rhum vieux que je viens de lui offrir et qui trône sur une table de pierre, aussi ambrée que la bouteille. Je lui tends le papier où j'ai consigné une suite de questions, en guise de balise à notre causerie. Il lit avec approbation, en sirotant son café. Au souvenir de ses mains, agitant ses lunettes comme une baguette de chef au moment de ses cours, je remarque qu'il n'a plus ses binocles. Les aurait-il cassés à force de les tortiller dans l'air, les faisant tournoyer sur leurs branches, tout en effeuillant ses notes? 


\section{Le choix de la discipline}

Il répète la première question et parait la tourner sept fois dans son silence avant de s'exprimer ainsi : «Pourquoi la géographie ? J'aurais aussi bien, en tout cas aussi, pu être historien. D'ailleurs, à la limite, je ne sais pas très bien quelle est la différence. Je me situe incontestablement dans la géographie, mais je revendique ma part d'historien. La chiquenaude est venue du "Concours général des lycées et collèges", cette vénérable compétition dont rêvaient tous les bons élèves, et du statut spécial qu'y avaient l'histoire et la géographie : que ce soit en première ou en terminale, on ne passait qu'une épreuve. Dans mon cas, c'était la géographie en première, l'histoire revenant aux terminales. L'année suivante, on inversa et j'eus encore droit à la géographie. De surcroît, il existait une épreuve transdisciplinaire, ouverte aux deux classes à la fois, et qui visait particulièrement à promouvoir l'étude de " nos colonies ", le concours de l'Union Française. Me présentant aux deux épreuves, j'y ai obtenu deux premiers prix. L'année suivante j'ai délaissé l'Union Française et me suis limité à la géographie, où j'eus encore un premier prix, toujours sur un sujet «tropical $»^{1}$. Donc j'ai passé le concours général deux fois en géographie "classique», une fois en " géographie de la colonisation » et obtenu trois premiers prix. Un record qui ne sera jamais battu puisqu'il n'y a plus d'Union Française. Pas de quoi, en fait, «rouler des mécaniques » : j'ai relu récemment ma copie de l'UF, parue au BO en 1953 : c'est bavard, et surtout terriblement conformiste : du travail de bon élève, modèle MRP. Lire de ces textes incite à ajuster son point de vue sur les copies de nos étudiants : que faisionsnous à leur âge?

5 J'ai raconté ailleurs ma décision de faire de la géographie et de la géographie africaine : motifs un peu sordides (être le premier dans son village plutôt que le second à Rome, en histoire, discipline plus noble). Mais apparition aussi d'une conscience politique et éthique : rompre avec le racisme ambiant dans le monde colonial. On voit là une logique. Elle est moins évidente dans ma décision de préparer Ulm au lycée Henri IV : il n'y avait pas de géographie au concours de la rue d'Ulm et Henri IV passait pour un lycée de joyeux fumistes. Le résultat n'a pas été mauvais, puisque j'ai «intégré » en 1957, à mon second concours, et me suis aussi bien amusé.

Dans ma décision, il y avait comme une volonté de rupture en douceur avec la famille. On était 5 enfants ; ma famille vivait correctement, mais on était quand même serrés les uns sur les autres. Je m'entends très bien avec tous mes frères et sœurs, mais il n'était pas toujours facile de voisiner dans la même chambre avec un frère de six ou sept ans quand vous en aviez seize. Spatialement, je ne m'éloignais guère : de la rue de Babylone à la rue d'Ulm il y a peu, mais j'étais, à vingt ans, indépendant.

7 Rue d'Ulm, les géographes n'étaient qu'un groupuscule: quatre, toutes années confondues, exceptionnellement deux dans ma promotion, Jacques Brun et moi. Notre mentor, Marcel Roncayolo, outre un cours annuel, jouait un rôle discret, mais précieux, d'orientation. Pour l'essentiel, moyennant une obligation de résultats, nous faisions à notre guise. Nous suivions nonchalamment des cours à l'Institut de Géographie, avec une préférence de fait pour ce qui était assez loin de nos orientations: Pierre Birot créant le monde à partir du Carbone, ça ne se ratait pas; pas plus que Dresch, improvisant de brillantes explications de cartes, ou la dialectique sans faille de Pierre George. Mais où trouver la tropicale ? il y eut au moins une année où, dans le certificat de géographie tropicale, il n'y avait aucun pays tropical au programme : le menu était 
Argentine et Maghreb. Il faut dire que le titulaire de la chaire, Charles Robequain, que je regrette de ne pas avoir connu, était gravement malade, "tué par ses médecins" disait son ami Gourou. Je connus celui-ci par mon père, qui allait l'écouter au Collège de France, et Sautter via Roncayolo.

8 - Jean Marie Théodat (JMT) : Mais quel hasard précisément vous a conduit en Afrique?

9 - Jean-Pierre Raison (JPR) : Il n'y pas de hasard: le Concours général me valut, outre une belle médaille de l'Assemblée de l'Union française (que j'ai toujours dans mon bureau) un billet d'Air France, un Paris Brazzaville, avec un retour par le Gabon, le Cameroun, le Tchad. J'ai coiffé un peu le casque colonial, et regrette d'avoir perdu ce couvre-chef symbolique.

10 - JMT : C'était en quelle année?

11 - JPR : C'était en 1953.

12 - JMT : Alors qu'est ce que la géographie a apporté à votre compréhension du monde?

13 - JPR : Elle m'a d'abord forcé à m'ouvrir sur le monde, sur les gens. J'étais très timide et quand je suis parti pour la première fois, faire du terrain dans le Boulonnais, interroger les exploitants, j'avais une forte trouille. J'ai même pris mon petit frère avec moi : «on va aller voir une ferme ». Comme ça, je ne pouvais pas reculer.

14 La géographie m'a donné une grille de lecture sur les relations avec l'extérieur, qui devenait relativement facile et commode à saisir. Je ne peux pas rester devant un paysage sans essayer de l'analyser. On n'a pas, comme en Histoire une trame de temps (1714 précède 1715). En géographie il faut mémoriser des lieux, des espaces; saisir des logiques, des systèmes territoriaux... Cette mémoire spatiale n'est pas donnée à tous : un de mes fils l'a bien plus que moi, et autant qu'un Malgache; curieusement, ce n'est pas le géographe des deux!

\section{L'importance du paysage}

15 - JMT : La géographie serait une appréhension du monde par le paysage ?

16 - JPR : Oui, je crois. Je restitue volontiers l'histoire à partir des paysages et l'inverse. J'aime pour cela la géographie historique. J'en ai fait un petit peu; des publications d'histoire j'en ai, et parmi les écrits que j'aime bien, il y a le texte que j'ai écrit pour les Annales sur l'espace, une manière d'anthropologie de l'espace : les Malgaches ne voient pas l'espace comme les Français et la vue qu'ils en ont, l'usage qu'ils en font, varient selon les catégories sociales, dans le cadre d'une "société géographique». Aucun élément d'espace n'est égal à un autre. Beaucoup de gens ne savent pas voir l'espace, et ne savent pas voir dans l'espace.

17 Dans le traitement géographique des paysages, on a toujours tendance à prendre de beaux paysages. Or la majeure partie des paysages ruraux en Afrique est esthétiquement médiocre, voire franchement déprimante. On vous montre toujours les chutes Victoria entre le Zimbabwe et la Zambie, mais la Zambie est un pays laid, sauvé par la gentillesse de ses habitants, un peu comme le pays mossi.

18 Laids ou beaux, les paysages végétaux ont un sens. Paul Pélissier, en ce domaine, a été mon maître. Il avait, au Sénégal, des paysages parmi les plus difficiles a priori à décrypter, d'autant qu'il n'usait pas de la photo aérienne. Les monts Mandara, le pays 
Kabyè, les Uluguru, ça plaît au géographe comme au touriste, mais le Sénégal arachidier....! Quand on est initié, on éprouve de grands plaisirs.

Connaissez-vous l'histoire de mes baobabs? Je faisais la route monotone de Nairobi à Mombassa. Comme on s'ennuie, on regarde le paysage. Au bout d'un certain temps, je m'avisai que des groupements de baobabs apparaissaient, peu avant les premières maisons des villages. J'étais à côté d'un géographe allemand spécialiste de l'Indonésie, un peu plus dépaysé que moi probablement, et je lui dis : « on va passer dans un village dans un ou deux kilomètres ", puis je l'ai fait une deuxième fois, une troisième fois.

20 - JMT : A chaque fois ça marchait?

21 - JPR : A chaque fois ça marchait. Alors il m'a dit « comment faites-vous »? Ce n'est pas compliqué, répondis-je "chaque fois que je vous dis, il va y avoir un village, si vous avez bien remarqué il y a un bouquet de baobabs, et tous les villages sont sur des sites à baobabs ». C'était, en fait, une zone de recolonisation par des gens qui étaient partis dans les monts Machakos et qui redescendaient occuper les plaines. Ils se fixaient en fonction des baobabs, parce que les baobabs sont signe de présence humaine antérieure. En l'occurrence le baobab est un signe de la fertilité des sols (plus riches en calcium). La problématique de Pélissier, on peut l'appliquer partant du Sénégal jusqu'au Kenya, en passant par le pays kabyè (Togo), avec la prudence d'usage, mais c'est un mode de lecture formidable.

22 Encore faut-il penser à se lancer dans cette archéologie de l'arbre. Je suis très redevable à Paul de m'avoir appris ça : incontestablement, ici, on ne voit pas ce qu'on ne connaît pas.

\section{Héritage et filiation intellectuelle}

23 - JMT : Quels ont été vos maîtres en géographie?

24 - JPR : J'en ai eu beaucoup, mais avec des statuts divers. Je mets à part mon père, à qui je dois énormément, mais la parenté fausse les rapports : on se disputait souvent, et la science masquait souvent autre chose.

Je n'ai jamais suivi un cours de Pélissier ; je l'ai connu après l'agrégation. Pour moi, il est un grand frère. On n'a pas après tout une différence d'âge formidable. Paul passe son temps à me dire qu'il est un vieil homme, mais son activité me confond, et nous n'avons après tout que 16 ans de différence, soit moins que je n'en ai avec mon plus jeune frère. Pour Sautter et Gourou, je ne parlerais pas de grand-frères, mais de maitres, avec des nuances, car ma familiarité à Gourou est surtout de l'écrit. Assurément, j'ai eu de la chance. Et ces maîtres ne se souciaient pas que de science : nos relations portaient sur l'ensemble de nos problèmes, de nos centres d'intérêt. Gilles était un type brillant, une intelligence, une culture tout à fait exceptionnelle, pas vraiment un virtuose du terrain, (on pouvait lui en faire croire ; à Paul, jamais). Gourou, c'est par la lecture que je l'ai connu. Je ne dis pas que j'ai tout lu mais... Un sacré tiercé tout de même : de grands scientifiques solidaires, une alliance réfléchie. Il n'y avait pas les mêmes rapports qu'aujourd'hui entre Alain Dubresson et moi : nous, nous sommes frères, et savons à peu près toujours d'avance comment l'autre va réagir. Lui ou moi, c'est pareil.

26 Je dois ajouter, sans faire de démagogie, que parmi les maîtres, il y a les étudiants aussi. Qui vous renvoient à une position, où vous êtes obligé de vous poser des questions sur 
la manière dont ils vous transcrivent et interprètent. Le besoin des étudiants, ou la manière dont les étudiants pigent ou ne pigent pas, sont très révélateurs. Il y a très peu d'articles que je n'aie pas testés en séminaire. Je les ai souvent considérablement modifiés ensuite, un peu au pif. On se dit, « non ça ne cadre pas là, ton raisonnement, il n'est pas bon ; à l'écrit ça ne se voit pas, parce que tu as le masque de l'écriture, mais là ça ne colle pas, tu vois bien que les gens ne suivent pas, ça décroche ».

27 - JMT : Il y avait une sorte de maïeutique inverse?

28 - JPR : Oui, je crois. Je crois effectivement beaucoup à ces flux qui passent, porteurs de connivence et de dynamisme, ce qu'on appelle le hasina à Madagascar. Le courant passe ou ne passe pas, l'expression est bonne ou non et on s'en rend bien compte y compris dans les grands amphis. Il m'arrivait quand je faisais des amphis de trois cents étudiants, je ne dis pas pour tous bien sûr, mais en suivant quelques étudiants repérés ici ou là, à distance, je me rendais compte qu'il y avait quelque chose qui n'allait pas. C'est peut-être très prétentieux, mais on ne peut pas travailler sur Madagascar, si on n'a pas ce genre de chose et si on n'a pas le regard (le regard est instrument de puissance ; il faut regarder, mais le faire avec circonspection pour ne pas humilier) et la maîtrise des points cardinaux. Ça c'est crucial, on s'en rend compte tout de suite. J'ai dû vous raconter les déplacements dans la maison malgache?

29 - JMT : Racontez encore, ce sera de nouveau un régal.

30 - JPR : Il faudrait reprendre l'article de ma femme. La maison malgache est orientée Nord Sud, et les fenêtres ne sont jamais à l'Est. On peut donner de ceci une explication déterministe : l'alizé en hiver. Moi, à la maison, dans mon bureau de $80 \mathrm{~m} 2$, j'avais une fenêtre à l'est et on se gelait. Le fond du problème est plus subtil : à chaque direction dans la maison correspond une place, soit pour les objets, soit pour les vivants. La place importante, c'est le nord-est, c'est le coin des ancêtres, où l'on gardera les statues et autres objets précieux de la famille, de la descendance ; à l'opposé, en diagonale, au sud-ouest, c'est celui des enfants, des esclaves, du menu fretin. Alors, normalement quand un vazaha (européen) arrive, on le met au nord-nord-est, un peu de biais, dans une position très inconfortable. Parce qu'on vous a mis sur une chaise, quand tous les autres sont assis par terre...

31 Dans une maison betsileo où je venais souvent, je me suis soudain aperçu que ma place avait changé : j'étais au sud-ouest, ma place normale d'ignorant qui vient s'instruire, alors qu'au début, bien sûr, on m'avait mis au nord-est. Je ne saurai jamais comment j'y suis arrivé, mais j'y étais bien!

32 - JMT : Mais est-ce que par exemple, ici dans cet appartement, au moment de l'aménager, vous avez tenu compte de cette géomancie malgache. Par exemple, vos endroits de prédilection, ici, correspondent-ils à cette injonction cardinale?

33 - JPR : Ici, on est au nord et en haut. Cela ne va pas plus loin. Mais je n'ai pas manqué de signaler à Augé, quand il était président de l'EHESS, que son bureau était au NE, à l'étage sommital!

34 - JMT : Votre position est donc également une position éminente?

35 - JPR : On est en haut, mais enfin, nous avons vécu à Tananarive en étant, pendant six ans, les plus hauts habitants de la ville. Ça vous marque, mais ce qui me marquait surtout, je ne m'en rendais pas compte, c'était la proximité du palais de la Reine. On était à deux cents mètres. Aujourd'hui qu'il a brûlé, je suis meurtri. 

demande urbaine d'immatriculations qui effraie les ruraux. Ce qu'il faut noter, c'est l'aspect général à Madagascar du problème foncier, parce qu'il y a aussi à l'heure actuelle des tas de demandes d'immatriculation de terres qui sont faites par la grande bourgeoisie malgache, notamment merina, dans un certain nombre de régions. Il y a un peu plus d'un an, dans une région riche d'Imerina, l'Itasy, il y a eu un procès fait à des villageois qui s'étaient rebellés contre les gendarmes qui voulaient les chasser de leurs terres, immatriculées sans qu'ils le sachent. Il y a eu un gendarme tué et au total 17 personnes ont été condamnées à mort. Elles n'ont pas, à ce que je sache, été exécutées. Le phénomène d'appropriation des terres, ou d'accaparement par les riches, est en train de se produire à grande échelle. Il y a un mouvement considérable de fermiers afrikaners s'installant au Mozambique. J'ai vu le même mouvement quand je travaillais en Zambie. J'étais au fin fond de l'ouest zambien, il y avait des Sud-Africains qui venaient prospecter et voir s'ils pourraient obtenir des concessions dans des coins intéressants. Ça date d'il y a plus de 10 ans.

\section{Le métier de géographe}

- JMT : Quel est votre rapport aux nouvelles orientations de la géographie?

45

: Je ne suis pas sûr que les géographes aient beaucoup gagné à être abstraits et chiffrés comme ils l'ont été. La géographie est aussi (surtout ?) poésie de l'espace. Il y a une chose qui m'inquiète beaucoup, c'est la disparition du terrain. La télédétection: j'aime beaucoup. Je ne sais pas en faire, je n'en ferai pas, mais ça m'intéresse, ça 
m'attire à condition de prendre ça comme une photo aérienne. J'aime le commentaire de poster. J'en ai fait l'essai avec Philippe Gervais-Lambony sur Harare, pour la Géographie Universelle (Reclus) : c'était extrêmement intéressant. Mais encore une fois : on trouve quand on connaît déjà !

46 J'aime la cartographie, mais je dois dire que pour la cartographie par ordinateur, dont j'ai été pionnier, je cale, je ne peux plus physiquement. Mais surtout on tombe dans trop de pièges. Il faut toujours bien définir ceux-ci. Le premier c'est la carte pressebouton, les cartes tournant autour de l'écart-type. Il faudrait d'abord demander aux étudiants ce que c'est qu'un écart type. L'expérience est probante : ils ne savent pas. On fait de belles cartes, sans savoir ce qu'elles signifient. J'ai travaillé à l'ancienne. J'ai perforé des cartons, établi des programmes simples... Quand on débutait, avec Hervé Théry, on notait les coordonnées, on les rentrait en machine. Faire une carte du Kenya, ça prenait quatre jours.

47 Le SIG : je m'y perds ; je croyais avoir compris, Jérôme Marie me dit que non, « tu n'as rien compris du tout, ce n'est pas ça ». Alors je regarde, convaincu en tout cas qu'il n'y a pas de SIG pertinent sans terrain.

48 - JMT : Vous m'avez dit récemment, au cours d'une conversation téléphonique, que vous vous sentiez dans la position du passeur, si vous avez passé quelque chose, qu'estce que c'est?

49 - JPR : C'est probablement une autre manière de répéter ce que j'ai déjà dit, il s'agit de savoir comment se sentir bien dans l'espace où l'on est, sensuellement, mais aussi intellectuellement. J'ai aussi le souci d'une belle écriture, ce que j'ai réussi à passer à quelques uns. Quant à me situer théoriquement, je ne suis pas un théoricien mais je me sens appartenir à une lignée de gens qui ont fait que la discipline existe. Je crois que c'est une espèce de continuité, je ne suis pas Sautter ni Pélissier. Les circonstances étaient telles qu'ils ont été des créateurs. Je ne suis pas sûr d'avoir été un créateur. Là où je l'ai été un peu, c'est dans les relations entre agronomes et géographes. Mais je n'ai pas eu grand mérite parce que les bons agronomes y sont venus, en quelque sorte, tout seuls. J'avoue que j'ai été sidéré quand j'ai vu Hubert Cochet rédiger sa HDR. Je le connaissais bien, je l'aimais bien, on a décidé que je le pilotais, mais je n'ai pas eu grand chose à piloter. Celui-là, il fait de la géographie comme un pommier fait des pommes !

50 - JMT : Quel effet votre thèse a-t-elle eu dans la connaissance de Madagascar?

51 - JPR : Ce livre est beaucoup trop gros. Je ne crois pas aux effets des grands écrits, par la taille, 1200 pages ça ne se lit pas. Tout le monde me le dit, à commencer par ma femme. On s'est disputé, avec Sautter, pour savoir si sa thèse était plus longue que la mienne, mais ce qui plaît et qui a de l'impact, il faut bien le dire, ce sont les petits articles courts, même quand ils sont mauvais, je dirais presque surtout quand ils sont mauvais, en tout cas « provocateurs "et écrits en anglais. Mais cela ne veut pas dire que ce soient des articles que l'on écrit très tôt, je crois qu'il faut au contraire beaucoup de temps pour faire bref. Voltaire le dit dans une de ses lettres, que je cite au hasard de ma mémoire : «Vous me pardonnerez la longueur de cette épître, je n'ai eu le temps de la faire plus courte » et je crois que c'est très vrai ». 


\section{NOTES}

1. «Les problèmes du peuplement blanc en Afrique centrale et australe » 